\title{
Economía social y solidaria y agroecología en cooperativas de agricultura familiar en Brasil como forma de desarrollo de una agricultura sostenible
}

\author{
Fabio Schwab do Nascimento \\ Ángel Calle-Collado \\ Rocío Muñoz Benito
}

RESUMEN: El objetivo de este artículo es comprender cómo la Economía Social y Solidaria (ESS) y la Agroecología pueden contribuir a la sostenibilidad económica, social, ecológica y política de la agricultura familiar en Brasil. Para ello, se expondrán sus dimensiones y las diferentes formas de obtener la viabilidad de determinadas explotaciones familiares. Como metodología de investigación se ha empleado el uso de indicadores de sostenibilidad creados por los autores para realizar los análisis de las experiencias de cooperativas de agricultores familiares en Brasil. Las cooperativas seleccionadas fueron las pertenecientes a la Asociación de Agricultores Ipê y Antonio Prado (AECIA) en la ciudad de Antonio Prado localizada en el estado de Rio Grande do Sul (Brasil) y la Cooperativa IRITUIA en la ciudad de Irituia en el estado de Pará (Brasil) como locus de la investigación. Los resultados de la investigación apuntan a que existen diferencias entre las cooperativas estudiadas en cuanto a la viabilidad productiva, social, política y económica tanto desde la ESS como desde la Agroecología, de manera que AECIA ofrece mejores valores en todos los atributos estudiados frente a la Cooperativa de Irituia.

PALABRAS CLAVE: Agroecología, Cooperativas, Brasil, Desarrollo sostenible, Economía Social y Solidaria.

CLAVES ECONLIT: D64, D63, 013, Q13.

Cómo citar este artículo / How to cite this article: SCHWAB, F., CALLE-COLLADO, A. \& MUÑOZ, R. (2020): "Economía social y solidaria y agroecología en cooperativas de agricultura familiar en Brasil como forma de desarrollo de una agricultura sostenible", CIRIEC-España, Revista de Economía Pública, Social y Cooperativa, 98, 189-211. DOI: 107203/CIRIEC-E.98.14161.

Correspondencia: Fabio Schwab do Nascimento, Instituto Federal do Para (Brasil) / Universidad de Córdoba, e-mail: Z32scnaf@uco.es; Ángel Calle-Collado, ISEC / Universidad de Córdoba, Angel.Collado@uco.es, y Rocío Muñoz Benito, Universidad de Córdoba, dt1muber@uco.es. 


\section{EXPANDED ABSTRACT}

\section{Social and solidarity economy and agroecology in family agriculture cooperatives in Brazil as a form of development of sustainable agriculture}

We urgently need to rethink solidarity and social economy strategies focused on endogenous development subjects and rural world. Social questions as food sovereignty, right to food, gender equality or small productions viability must be addressed and evaluated, among other reasons, because of their current importance in the political agendas of organisms such as the FAO or the Intergovernmental Panel on Climate Change (IPCC). These institutions have been warning for decades about the social and environmental need of turning the goal of food sovereignty into reality.

The concept of Food Sovereignty was defined in 1996 during the FAO Summit on the Right to Food in Rome. There, the Vía Campesina movement defined Food Sovereignty as a social and political concept essential to build bridges between the agroecological and social and solidarity economy perspectives. This movement referred to the sovereignty of communities, peoples and consumers in order to decide what to eat, what to produce and how the territorial development must be according to its limitations and endogenous characteristics. This concept proposed a higher visibility for the peasant movement, taking into consideration the lack of attention paid as an intermediary agent in international forums and decision-making spaces on the World Trade Organisation or the development policies of other international institutions.

With the proposal of an agenda focused on concepts (endogenous development, Food Sovereignty), we need located agri-food systems which work from the logics of community economies, social-solidarity economies and caring economies. We consider that our monitoring and researching projects must reflect this need of theoretical and methodological frames. These ones combine inputs from different fields of social and environmental sustainability.

Because of these reasons, this paper aims to understand how the Social and Solidarity Economy (SSE) as well as the Agroecology can contribute to the economic, social, ecological and political sustainability of family agriculture in Brazil. For this purpose, the dimensions and different ways for viability of selected family exploitations will be exposed. The study is about collecting how the ESS

and the Agroecology dialogue on many factors related to sustainability in the production system and peasants' organisation. 
One of the commonest problems of many SSE experiences is how environmental protection remains at a second stage, if compared to democracy or redistribution issues. Therefore, by adopting Agroecology, the current agroecosystems artificialisation model could be changed. This system would be more suitable for social and ecological reproduction through new proposals of technical, ecological, agronomical and social production. Via this production, a sustainable management of natural resources could be achieved avoiding degradation of the environment.

Another question to deal in this paper is the role developed by the short circuits of commercialisation in the promotion of agroecological strategies conceived at the same time as an answer from the perspective of social and solidarity economy. Short circuits of commercialisation are essential elements, especially for peasants and for the endogenous rural development, in order to link again production and consumption to principles of proximity, reliance, and sustainability. The adoption of short circuits as a strategy for territory sovereignty and a defence is a way of guaranteeing the economic aspects as well as the social ones in Agroecology.

Our case studies are based in representative experiences of different ways of commercialisation in the Northern and Southern regions of Brazil: Associação dos Agricultores Ecologistas de Ipê y Antonio Prado (Ecologist Farmers Association of Ipê and Antonio Prado, AECIA) and the Cooperativa de Irituia (Cooperative of Irituia). The AECIA is in the Brazilian province of Rio Grande do Sul, Northeast region, in the municipalities of Antonio Prado and Ipê. These present an intermediate level of capitalisation, where the production systems integrate vegetal and animal productions, outlining the conventional and ecological fruticulture (grape, apple, peach), ecological horticulture, dairy cattle, marking livestock, pigs, birds and corn. The Cooperative of Irituia is in the Northern region of Brazil, province of Pará, municipality of Irituia, in the Northeast mesoregion. Its population mainly lives in the rural environment. Its local economy, which is based in agriculture and wood extraction and transformation, is mainly composed of family peasants that practice subsistence farming. Both belong to the tradition of family agriculture in Brazil, but they present a different approach in their ecological, community or economic perspective where they interact.

Regarding the method for the case study, we have applied a qualitative approach through several social research techniques, some of them are explicative (interviews, experts panel), other are more inductive (survey based in three dimensions: Agroecology, New Commons and SSE) and other are more descriptive (secondary sources, interviews to key informers). With all this material, we have made a proposal of social-economic, social-cultural and productive sustainable indicators. In this way, we can test the sustainability approach of these initiatives in the long term. 
Results of the research point to the existence of differences between the analysed cooperatives regarding productive, social, political and economic viability, not only from the SSE but also the Agroecology. Because of this, the AECIA offers better values in all the studied items instead of the Cooperative of Irituia. At a theoretical level, this work demonstrates a more complex approach to the short circuits in the agri-food channels. Furthermore, from a common agroecological and SSE point of view, those are introduced as an umbrella for democratisation of our agri-food systems, meanwhile defending the right to food as well as a sustainable and healthful production suitable to the physical and cultural context.

KEYWORDS: Agroecology, Cooperatives, Brazil, Sustainable development, Social and Solidarity Economy. 


\section{Introducción}

La Agricultura Familiar en Brasil ha sufrido diversos problemas económicos, sociales y ambientales relacionados con la adopción de un modelo de agricultura empresarial capitalista que promueve un sistema de producción basado en el uso de pesticidas y productos químicos (Boeckmann y otros 2014). Podemos encontrar ciertos paralelismos entre la situación de Brasil y la actual crisis del medio rural en España (Aparecida y otros 2014).

La importancia de trabajar con el tema que es objeto de este estudio es la necesidad de aportar nuevas propuestas que aúnen sostenibilidad social, económica y ambiental en un contexto problemático para la agricultura familiar: los mercados globales suponen una merma de ingresos y de capacidad de decisión en los sistemas agroalimentarios mundiales y locales; se extiende la amenaza de migraciones y despoblación del medio rural; el cambio climático supone la necesidad de estudiar cómo relocalizar economías, clave para la pequeña agricultura.

Esta investigación tiene como objetivo comprender de qué forma podrían contribuir la ESS y la Agroecología a la solución de esos problemas y desarrollar otra agricultura en Brasil, basada en los principios de sostenibilidad social y ambiental de los agricultores familiares.

Como metodología de investigación se ha empleado el uso de indicadores de sostenibilidad creados por los autores para realizar los análisis de sostenibilidad de dos experiencias de cooperativas de agricultores familiares en Brasil. Las cooperativas seleccionadas fueron las pertenecientes a la Asociación de Agricultores Ipê y Antonio Prado (AECIA) en la ciudad de Antonio Prado localizada en el estado de Rio Grande do Sul (Brasil) y la Cooperativa IRITUIA en la ciudad de Irituia en el estado de Pará (Brasil) han sido nuestro locus de la investigación.

El análisis, además de una tabla de indicadores, incluye un cuestionario cuya finalidad es evaluar los resultados concretos de las experiencias con base en las siguientes cuestiones: ¿Cuáles son las fortalezas adquiridas con la adopción de la Agroecología y la ESS?; ¿Cuáles son los principales problemas a los que aún se enfrentan?; ¿Cuáles son las perspectivas de futuro respecto al modelo de producción?

En primer lugar, se realizó un análisis de los diferentes conceptos de Agricultura Familiar, ESS y Agroecología. A continuación, se elaboró un histórico de la ESS y Agroecología en Brasil. En la tercera parte se discutieron las dimensiones de la sostenibilidad económica, social, política y ecológica de la Agroecología y la ESS. Posteriormente se realizó una evaluación de los indicadores de sostenibilidad de la ESS y la Agroecología dentro de las dimensiones propuestas en cada una de las 
experiencias. Por último, en las conclusiones, se pretende demostrar cómo el éxito de esas experiencias puede estimular la adopción de la ESS y de la Agroecología en las cooperativas de agricultura familiar en Brasil como modelo de desarrollo frente a otras alternativas.

\section{Análisis de conceptos}

En primer lugar, analizaremos el marco teórico de la Agricultura Familiar, la ESS y la Agroecología para comprender mejor lo que cada una puede aportar para el desarrollo de la agricultura.

\subsection{Concepto de Agricultura Familiar}

Tanto en países considerados desarrollados como en vías de desarrollo la agricultura familiar es la realidad más presente en cuanto a número de personas implicadas, producción de frescos y abastecimiento de mercados locales. La agricultura familiar es un concepto amplio que comprende, obviamente dado su adaptación al contexto cultural y territorial, realidades muy heterogéneas (Buainain, 2003)

La Organización de Naciones Unidas para para la Alimentación y la Agricultura define la agricultura familiar:

"La agricultura familiar (incluidas todas las actividades agrícolas basadas en la familia) es un medio de organizar la producción agrícola, forestal, pesquera, pastoral y acuícola que es administrada y operada por una familia, y depende principalmente de la mano de obra familiar de mujeres y hombres. La familia y la granja están vinculadas, coevolucionan y combinan funciones económicas, ambientales, sociales y culturales" (FAO y IFAD, 2019: 10).

En Brasil existen conceptos distintos para definir lo que se entiende por agricultura familiar. Algunos utilizan para definirla parámetros económicos, normativos y otros de tipo social. En la normativa brasileña, dentro del ámbito de las políticas públicas, según la Ley 11.326 del año de 2006 se considera agricultor familiar o Emprendedor Familiar Rural aquel que tenga el máximo de cuatro módulos fiscales ${ }^{1}$, utilice predominantemente mano de obra de la propia familia en las actividades económicas de la propiedad, tenga porcentaje mínimo de la renta familiar que venga de las actividades económicas de la propiedad y gerencie su propiedad con su propia familia.

1.- Módulos fiscales - es una unidad de medida en hectáreas establecidas por el Instituto Nacional de Colonización y Reforma Agraria (INCRA). 
Otro concepto económico es el de Neves (2007), que entiende la agricultura familiar como una forma de organización y de producción en que, al mismo tiempo que ella es propietaria de los medios de producción también ejecuta las actividades productivas.

En el análisis sociológico se puede clasificar la agricultura familiar conforme Van der Ploeg (2010) en dos tipologías, una campesina y otra empresarial. La primera se caracteriza por ser diversificada y la producción está destinada tanto para el mercado cuanto para para la familia. En la segunda, la producción es completamente especializada y totalmente destinada para el mercado.

\subsection{Concepto de ESS}

La ESS forma parte de una rama de la economía social que critica el modelo capitalista y propone una democratización de la economía a través de la organización de la sociedad de manera que pueda satisfacer las necesidades de sus miembros y también su desarrollo (Barea, 1990; Calle-Collado y Casadevente, 2015; dos Santos et al., 2017).

Para autores como Laville (1994), Laville y Roustang (1999), Ortiz Roca (2001), Lechat (2002), Tauile y Debaco (2002) Singer (2003) la ESS se ajusta más a la idea de organización de las actividades económicas por medio de relaciones de solidaridad, reciprocidad, autoorganización, articulación, autogestión, cooperación e inclusión, evitando así los problemas económicos, sociales y políticos generados por la economía capitalista de corte neoliberal. Otro concepto muy similar es el de la ONU (2014), la cual define la ESS como un enfoque económico que favorece la descentralización y el desarrollo local que se rige por valores éticos como la solidaridad, el comercio justo, la simplicidad voluntaria y el Buen Vivir.

Entendemos que la ESS va más allá de los planteamientos estrictamente económicos, no obstante, existen múltiples estudios que avalan la capacidad de las empresas y modelos de economía social para mejorar el bienestar de las personas (Cabanes y Gómez, 2014) y el desarrollo de los territorios, especialmente en zonas rurales (Mozas y Bernal, 2006; Daza y Dangla, 2013; Gómez y De Souza 2013). Estos conceptos que van más allá del enfoque estrictamente economicista, la definen como un sistema económico, político y social que, por medio de aproximaciones, podemos englobar en una Economía crítica, tales como el Ecofeminismo, los Nuevos comunes, la Economía de los cuidados o las perspectivas de decrecimiento económico con justicia, entre otras, (Pérez de Mendiguren et al., 2008; Cuéllar y Calle-Collado 2009; Carpintero y Riechmann, 2013; Calle-Collado y Casadevente, 2015, Alonso y Piñero, 2015, Calle-Collado et al, 2017). 


\subsection{Concepto de Agroecología}

La Agroecología no consiste solamente en el sistema productivo de la finca, monte o explotación ganadera. Es un sistema directamente relacionado con la sostenibilidad productiva y el derecho a la alimentación. Siguiendo los trabajos clásicos en la materia de Sevilla y Woodgate (1997), Guzmán et al. (2000) y Sevilla (2011) la Agroecología comprende diversas formas de acción social colectiva enfocadas a la satisfacción amplia de necesidades (culturales, políticas o de bienestar personal) ligadas a la construcción de nuestros Sistemas Agroalimentarios ${ }^{2}$ (SAG). Se plantea de forma articulada desde la finca, como forma de organización comunitaria incluyendo la importancia de la cuestión local, es decir, no dejando que la propiedad quede aislada, tal y como ocurre en el actual modelo agroalimentario. Además, trabaja con el empoderamiento de los agricultores y consumidores donde los conocimientos de los campesinos 0 indígenas son valorados desde su aportación a la biodiversidad. Caporal y Costabeber (2004) entienden la Agroecología como un campo de conocimiento interdisciplinario que utiliza ciencias naturales y sociales, además de valorar el conocimiento de los agricultores. Esta forma diferente de hacer agricultura promueve, según ellos, niveles crecientes de sostenibilidad en sus múltiples dimensiones. En relación con este concepto, añade al anterior esta visión de que la unión de los conocimientos es necesaria para conseguir un sistema agroalimentario sostenible.

Por su parte, para Cuéllar y Calle-Collado (2009) la Agroecología es una aproximación a la producción agrícola, y al sistema agroalimentario en general, basándose en un enfoque participativo de desarrollo endógeno, en aras de lograr una sostenibilidad ecológica y también una democratización radical de quién decide cómo nos alimentamos.

\subsubsection{Agroecología y ESS en Brasil: una perspectiva histórica}

En la década de los 80 , surgen en todo Brasil una serie de experiencias e iniciativas a partir de los movimientos sociales y Organizaciones no Gubernamentales (ONG) como CAPA (Centro de Apoyo al Pequeño Agricultor) Centro Ecológico Ipe ${ }^{3}$, CETAP (Centro de Tecnologías Alternativas Populares) y Fundación Gaia, que adoptan modelos menos agresivos para el medioambiente y más adecuados para las condiciones económicas y sociales de la agricultura familiar. Esas ONG utilizan el término de "tecnologías alternativas" para definir un tipo de agricultura opuesta al modelo de la revolución verde, que pretendía encontrar junto a los pequeños agricultores, formas de agriculturas más adecuadas a sus necesidades rescatando conocimientos perdidos por estos.

2.- Elementos que componen la cadena alimentaria, desde materias primas y producción, hasta transformación y distribución, pasando por las políticas públicas y las culturas alimentarias que condicionan la anterior cadena

3.- ONG que asesora a las organizaciones de agricultores familiares en la producción, procesamiento y comercialización de alimentos orgánicos. 
A finales de la década de los 80 con la publicación del libro Agroecología: As Bases Científicas da Agricultura Alternativa de Miguel Altieri se comienza a adoptar, por parte de los movimientos sociales, el nombre de Agroecología para referirse a lo que antes se denominaba agricultura alternativa.

La Agroecología como alternativa al modelo de agricultura convencional para la Agricultura Familiar tiene su mayor impulso a partir de finales de la década de los 90 cuando en Rio Grande do Sul el gobierno de Olivio Dutra la emplea como una de las políticas públicas para la Agricultura Familiar.

A partir de los años 2000 la Agroecología pasa a ser una política pública destinada a la Agricultura Familiar a nivel federal por medio de varios programas gubernamentales. En el año 2012 se inicia, por medio de un proyecto de ley 4685/2012, un cambio que tiene como finalidad crear una política nacional de ESS. No obstante, hasta el año 2017 no se dispone de una normativa sobre esta materia. La primera norma que propone directrices y objetivos para la Política Nacional de Economía Solidaria, con el objetivo de fomentar esa economía y el trabajo asociado y cooperativo fue la ley 137/2017. Los diferentes gobiernos que se han sucedido han restado importancia a estos temas, de manera que la ley no se aplicó por el gobierno de Michel Temer y la perspectiva es que con el gobierno Bolsonaro pueda llegar incluso a eliminarse.

La evolución de la ESS en Brasil puede ser dividida en cuatro grandes etapas conforme la tabla 1 (basada en Gaiger, 2001 y 2003; Bialoskorski, 2004; Fórum Brasileiro de Economía Solidaria, 2008; Gonçalves, 2013; Silva y Carneiro, 2016; Silva et al, 2014; Costa y Jesús, 2017). 


\section{Tabla 1. Evolución de la ESS en Brasil}

\begin{tabular}{|c|c|c|}
\hline Etapa & Periodo & Características \\
\hline 1 & $\begin{array}{l}\text { Inicia en } 1980 \\
\text { hasta } 2000\end{array}$ & $\begin{array}{l}\text { - Primeras experiencias a nivel gubernamental en la ciudad de São Paulo y Estado de } \\
\text { Rio Grande do Sul. } \\
\text { - Paul Singer }{ }^{4} \text { comienza a trabajar con ESS en Brasil. } \\
\text { - Surgen diversas experiencias de la sociedad civil en diversas áreas trabajando la ESS } \\
\text { como la creación y constitución de cooperativas, bancos y experiencias cuya base es la } \\
\text { economía solidaria. } \\
\text { - Comienzan a surgir también en el medio rural experiencias vinculadas a ESS como } \\
\text { forma de alternativa para la resolución de los problemas generados tanto por la crisis } \\
\text { económica como por la agricultura convencional. }\end{array}$ \\
\hline 2 & 2001 a 2006 & $\begin{array}{l}\text { - Fase de ampliación y difusión de ESS y sus articulaciones. } \\
\text { - } \quad \text { Creación de la Secretaria Nacional de Economía Solidaria. } \\
\text { - } \quad \text { Puesta en marcha de políticas públicas del gobierno de Lula da Silva. }\end{array}$ \\
\hline 3 & 2006 a 2011 & $\begin{array}{l}\text { - Discusiones que llevaron a la necesidad de replantear la fusión y las estrategias de } \\
\text { ESS entre el Fórum Brasileiro de Economía Solidaria (FBES) y el gobierno. } \\
\text { - Se incorporan las razas, el medioambiente, las mujeres y las varias luchas sociales, } \\
\text { lucha por la reforma urbana, por la gestión responsable de los residuos urbanos, la } \\
\text { lucha por la equidad de género y otras. } \\
\text { - El FBES deja de actuar como interlocutor entre gobierno y sociedad civil en materia de } \\
\text { ESS. } \\
\text { - Reestructuración interna de FBES. }\end{array}$ \\
\hline 4 & $\begin{array}{l}\text { A partir de } 2012 \\
\text { hasta ahora. }\end{array}$ & $\begin{array}{l}\text { - FBES trabaja con una idea de proyecto político de economía solidaria para la sociedad } \\
\text { brasileña; } \\
\text { - Programas del gobierno de Dilma Roussef en materia de ESS como, por ejemplo, la } \\
\text { erradicación de la pobreza y el "emprendimiento". } \\
\text { - La entrada del Gobierno de Temer conlleva la pérdida de importancia de la ESS a nivel } \\
\text { de política pública del gobierno } \\
\text { - Con el principio de gobierno de Bolsonaro las políticas de ESS comienzan a ser retira- } \\
\text { das de las políticas públicas de Brasil. }\end{array}$ \\
\hline
\end{tabular}

FUENTE: Elaboración propia.

En relación con datos en el último censo sobre ESS en Brasil elaborado por el Departamento Intersindical de Estadística y Estudios Socioeconómicos (DIEESE) de 2014, existían en torno a 19.708 proyectos empresariales, los denominados "Emprendimientos Económicos Solidarios" (EES) (DIEESE, 2015).

4.- Paul Singer fue un economista de origen austriaco impulsor de la economía social y solidaria y referencia intelectual en América Latina. Estudió economía en la Universidad de São Paulo, donde posteriormente obtuvo un doctorado en Sociología. Posteriormente estudio demografía en la Universidad de Princeton. Profesor de la Universidad de São Paulo, aunque fue docente en varias universidades de Brasil y a nivel internacional. Cofundador del Centro Brasilero de Análisis y Planificación (CEBRAP), un reconocido centro de investigación brasileño. Del 2003 a 2016 fue Secretario Nacional de Economía Solidaria de los gobiernos de los presidentes da Silva y Rousseff. 
2.3.2. La mirada de la Agroecología y de la ESS para alcanzar la sostenibilidad económica, social, política y ecológica

La ESS y la Agroecología dialogan sobre muchos factores relacionados con la búsqueda de la sostenibilidad en el sistema de producción y de la organización de los agricultores. Una de las formas de establecer ese diálogo es la adopción de prácticas y tecnologías de convivencia con la naturaleza y de autonomía frente a las empresas que producen materias primas (Nobre, 2015).

De acuerdo con Calle-Collado et al. (2017), uno de los problemas que presentan muchas experiencias de ESS es que el cuidado ambiental queda relegado a un segundo plano, frente a cuestiones como democracia o redistribución. Por lo tanto, con la adopción de la Agroecología, se produciría el cambio del modelo actual de artificialización de los agroecosistemas, de manera que este sistema sería más adecuado para la reproducción social y ecológica a través de nuevas propuestas de formas de producción técnicas, ecológicas, agronómicas y sociales. Por medio de esta producción se conseguiría obtener una gestión sostenible de los recursos naturales, evitando así su degradación.

La implementación de sistemas agrícolas agroecológicos, potencia los comportamientos ecológicos naturales que son beneficiosos para el ecosistema además de incidir en su conservación y regeneración a través del máximo aprovechamiento de los recursos internos y externos potenciando las sinergias positivas en la producción de alimentos (González Calo, 2017).

El comercio justo se produce cuando la Agroecología aprende de la ESS en lo relativo a la organización de los agricultores a través de la autogestión y la democratización de sus experiencias organizativas tales como asociaciones, grupos o cooperativas. Tanto para la ESS como para la Agroecología la viabilidad y sostenibilidad también se potencian por medio de la organización de los agricultores y de los consumidores en grupos, asociaciones o cooperativas.

La adopción de un cooperativismo social agroecológico, que no es solamente un asociativismo económico entre los participantes, va más allá, siendo un modelo de producción, consumo, autogestión, independencia, organización democrática, horizontalidad en la toma de decisiones, autoformación e integración al territorio (tanto de los productores como de los consumidores). Además, esa organización social de los agricultores familiares también fomenta la cooperación, solidaridad, igualdad, libertad, autoorganización y democratización entre ellos. Es por ello por lo que la búsqueda de una agricultura participativa por medio colectivo, cooperado, autogestionado, tutelado o institucionalizado contribuye al desarrollo de una democracia participativa y radical.

Las redes de producción o de consumo provocan que la economía local sea incentivada a través de la valorización del trabajo de los productores locales. Ellas son responsables de que los ingresos se queden en ámbito local formando así una cadena de entrada de recursos y trabajos. Lo que permite mejorar la situación económica de las personas e incluso erradicar la pobreza, fortalecer la 
organización de base, preservar el medioambiente y restaurar las relaciones dentro de la comunidad de la solidaridad a través de la ESS y la Agroecología. Estas relaciones son tanto de los actores del campo como de la ciudad a través de las conocidas como "alianzas de pequeños" que son microespacios públicos autónomos o de proximidad que permiten el intercambio (Fraisse, 2004; Laville, 2004 Orozco, 2004; Aguiar, 2009).

Otro punto en el que convergen la Agroecología y la ESS es en lo relativo a la comercialización. Este es uno de los factores que causa falta de sostenibilidad económica y que enfrenta a la agricultura familiar con los mercados capitalistas. Casares y Rebollo (2005) Montiel y Calle-Collado (2010) y Sevilla et al. (2012) entre otros, defienden que esta comercialización debe realizarse empleando los canales cortos de comercialización (CCC 5 ).

Los CCC son para los agricultores y para el desarrollo rural elementos clave para volver a vincular la producción y el consumo como principios de proximidad, confianza y sostenibilidad. La adopción de los circuitos cortos como estrategia de soberanía y defensa del territorio, es una manera de garantizar tanto las dimensiones económicas como las sociales de la Agroecología.

Sin embargo, con relación a la certificación, encontramos diferencias entre las dos; en la Agroecología esta sirve para garantizar que la producción es agroecológica u orgánica, mientras que en la ESS garantiza que está dentro de sus principios. No obstante, la certificación no garantiza realmente que el producto 0 la experiencia está realmente produciendo un cambio en sus sistemas, por ejemplo, en el caso de la Agroecología según Bye y Schmidt (2001), puede generar una estandarización y calificación para un nicho de mercado. Otro caso es en relación con la certificación de comercio justo como Fairtrade ${ }^{6}$ que muchas empresas utilizan como estrategia de diferenciación frente a sus competidores. En este último caso, las empresas se vinculan a la ESS como estrategia de marketing, pero en realidad solamente una parte de sus operaciones tienen algún punto en común con la ESS.

5.- Canales de comercialización donde el papel de minorista y de mayorista se realiza por un único agente, siendo este el único eslabón en la cadena de valor entre el agricultor y el consumidor.

6. - Terminología en ingles de la Federación Internacional de Comercio Alternativo. 


\section{Metodología}

La investigación se realiza en el seno de dos organizaciones agrarias en Brasil: Asociación de los Agricultores Ecologistas de Ipê y Antônio Prado (AECIA) y la Cooperativa de Irituia. La razón por la que se eligieron estas organizaciones radica en su capacidad de mostrar las diferentes formas de la Agroecologia y ESS existentes, tanto en el norte como en el sur de Brasil; y en la pluralidad que presentan. Por lo tanto, a través de estas organizaciones, podremos entender la dinámica agroecológica y de la ESS que operan en la agricultura familiar brasileña.

Como estudios de caso elegimos experiencias representativas de distintas formas de comercialización en las regiones norte y sur de Brasil: Asociación de los Agricultores Ecologistas de Ipê y Antonio Prado (AECIA) y Cooperativa de Irituia. AECIA está localizada en la provincia de Río Grande do Sul en Brasil, en la región nordeste en los municipios de Antonio Prado e Ipê. La agricultura es familiar y bastante diversificada. Presenta un nivel medio de capitalización, donde los sistemas de producción integran producción vegetal y animal, destacándose la fruticultura convencional y ecológica (uva, manzana, melocotón), horticultura ecológica, el ganado de leche y de corte, cerdos, aves y maíz. Por su parte la Cooperativa de Irituia se encuentra en la región norte de Brasil en la provincia del Pará en el municipio de Irituia en la mesoregión nordeste y su población reside mayoritariamente en el medio rural. Su economía municipal se basa en la agricultura y en la extracción y la transformación de la madera y se compone principalmente de agricultores familiares que practican la agricultura de subsistencia. Ambas son deudoras de tradiciones de agricultura familiar en Brasil, pero con una aproximación diferente en su perspectiva ecológica, comunitaria o de economías con las que se relacionan en su entorno más cercano.

Con respecto al método de estudio para cada caso, se ha seguido principalmente un enfoque cualitativo a través de diversas técnicas de investigación social, algunas explicativas (entrevistas, panel de personas expertas), otras más inductivas (cuestionario realizado sobre tres dimensiones desde la Agroecología, Nuevos comunes y la ESS) y otras más descriptivas (fuentes secundarias, entrevistas a informantes clave). El estudio de caso es un método adecuado cuando se tiene una problematización del tema que se inicia con un "por qué" o "cómo" (ocurre esto o aquello); y cuyos objetivos y marco teórico se encuentres perfectamente identificados (Meirinhos y Osorio, 2010). 
La investigación se dividió en tres etapas. En la etapa inicial se realizó el análisis bibliográfico mediante búsquedas en bases de datos, de libros, revistas y periódicos nacionales e internacionales especializados en el tema ${ }^{7}$. En la segunda fase del trabajo se realizó una observación directa y cualitativa empleando las siguientes estrategias: observación participante, entrevistas semiestructuradas, reuniones y conversaciones. En el caso de las entrevistas semiestructuras si trabajo con 12 agricultores como muestra representativa de las experiencias, lo que corresponde aproximadamente al $20 \%$ de los participantes en cada una de ellas. El objetivo era comprender las dinámicas de la acción colectiva desde la perspectiva de los propios actores, siguiendo el enfoque clásico de Alonso y Benito (1998). La tercera y última etapa tuvo como base un abordaje cuantitativo y cualitativo para proponer por medio de todas las informaciones de las etapas anteriores, una sistematización de los resultados y el análisis de los datos obtenidos.

Fruto de las dos últimas etapas se construyó un sistema de indicadores que consideramos una aproximación inicial al análisis de los circuitos de comercialización desde la perspectiva de los Nuevos Comunes y de la ESS, y cuyo esquema general puede verse en Nascimento (2019) y Calle Collado e Nascimento (2019).

\section{Análisis de los resultados}

Teniendo como base una perspectiva teórica dentro de la investigación y en las observaciones hechas por los expertos, se creó una herramienta (basada en indicadores de sostenibilidad) para poder evaluar de una forma más concreta la aportación de la Agroecología y de la ESS a la experiencia. El trabajo completo puede ser encontrado en la tesis doctoral de Nascimento (2019).

En este artículo se analiza si la incorporación de la Agroecología y ESS está contribuyendo a la sostenibilidad económica, social, ecológica y política en esas experiencias. En lo relativo a la Agroecología definimos varias dimensiones como características fundamentales para entender de qué forma está contribuyendo para la sostenibilidad.

Para una mejor compresión en este artículo se ha modificado la siguiente tabla de criterios que aparece recogida en la tesis doctoral de Nascimento (2019).

7.- Los informes anuales sobre agricultura familiar, campesinado o circuitos cortos de comercialización facilitados por organizaciones como FAO, IFOAM o el IPCC son una buena introducción al tema, a escala mundial y por diferentes territorios. 


\section{Tabla 2 Criterios aplicados a Agroecología}

\begin{tabular}{|l|l|l|c|c|}
\hline Criterio & Dimensión analizada & Indicadores & AECIA & Irituia \\
\hline 1 & $\begin{array}{l}\text { Relación de materias primas } \\
\text { propias / materias primas externas } \\
\text { colaborativas / externas capita- } \\
\text { listas }\end{array}$ & $\begin{array}{l}\text { (0) NS/NC; (1) mayor parte o la totalidad del origen } \\
\text { es externo capitalista; (2) externo capitalista, poco } \\
\text { externo colaborativo o propio; (3) propio y externo; } \\
\text { (4) mayor parte propio y poco externo; (5) solamente } \\
\text { propio }\end{array}$ & 2 & 4 \\
\hline 2 & $\begin{array}{l}\text { Oferta de especies/variedades } \\
\text { locales }\end{array}$ & $\begin{array}{l}\text { (0): NS/NC; (1): no tiene; (2): puntual o eventualmen- } \\
\text { te; (3): pocas; (4): varias; (5): todas }\end{array}$ & 4 & 4 \\
\hline 3 & $\begin{array}{l}\text { Grado de proceso de transición } \\
\text { agroecológica }\end{array}$ & $\begin{array}{l}\text { (0): NS/NC; (1): producción convencional; (2): parte } \\
\text { de la producción es convencional, y parte agroecoló- } \\
\text { gica, o en transición (3): reducción y racionalización } \\
\text { de las prácticas convencionales; (4): sustitución de } \\
\text { materias primas químicas por otros de origen biológi- } \\
\text { co; (5): gestión de la biodiversidad y rediseño de los } \\
\text { sistemas productivos. }\end{array}$ & 5 & 2 \\
\hline 4 & Grado de diversificación & $\begin{array}{l}\text { (0): NS/NC; (1): ninguna diversificación (monocul- } \\
\text { tivo); (2): poca; (3): media; (4): alta; (5): totalmente } \\
\text { diversificada. }\end{array}$ & 3 & 3 \\
\hline 5 & Tipo de certificación adoptada & $\begin{array}{l}\text { (0): NS/NC; (1): ninguna; (2): propia; (3) OCS8; (4): } \\
\text { participativa; (5): auditoría }\end{array}$ & 3 y 4 & 3 \\
\hline 6 & Tipos de canales utilizados & $\begin{array}{l}\text { (0): NS/NC; (1): CCC; (2): mayor parte CCC; (3): } \\
\text { CCC y CLC; (4): mayor parte CLC; (5): CLC }\end{array}$ & 3 & 3 \\
\hline
\end{tabular}

FUENTE: Nascimento, 2019.

En relación con la experiencia de la $\mathrm{AECIA}$, los resultados muestran que la sostenibilidad adquirida se debe al hecho de disponer de un sistema de producción agroecológica bastante desarrollado, visto que, todas las propiedades ya producen de forma agroecológica. Pero aún presenta problemas debido a la dependencia de materias primas y productos externos para poder mantener una producción agroecológica y a la dificultad de acceso a mano de obra para poder mantener una diversidad en la producción. Un aspecto positivo es que existe una diversidad de especies y variedades locales que son adaptadas a la región, haciendo que se obtenga una biodiversidad productiva a pesar de los problemas relacionados con las materias primas.

8.- Organic Content Standard o estándar de contenido orgánico (OCS por sus siglas en inglés) se aplica a cualquier producto no alimentario que contenga un 95-100\% de materia orgánica en su composición. Verifica la presencia y cantidad de materia orgánica en un producto a la vez que controla el flujo de las materias primas desde su origen hasta el producto final.

9.- Los circuitos largos o CLC son líneas de comercialización en las que participan muchos actores, que pueden o no incorporar valor al producto final. 
Toda la producción ha sido certificada tanto por OCS como por la red ECOVIDA ${ }^{10}$. La comercialización se realiza por medio de CCC y CLC gracias a la visión estratégica de la experiencia que plantea estrategias de mejora basadas en la diversificación de canales de comercialización frente a la dependencia que supone emplear un único canal de distribución. En cifras actualmente este reparto de valores está en 30\% mercadillos de agricultores, 35\% grandes cadenas de supermercados (Zafari, Super Nosso), 35\% mercados menores (supermercados regionales, tiendas especializadas, otros).

En el trabajo de Schultz (2006) la experiencia facturaba aproximadamente 220.000 euros ( $\mathrm{R} \$ 11$ $1.000 .000,00)$ mientras que en 2018 este valor alcanzó los 330.000 euros $(R \$ 3.000 .000,00)$ según los datos proporcionados por COOPAECIA, lo que supone un aumento del $300 \%$ en la renta de los agricultores de la experiencia en ese periodo.

En la experiencia de la Cooperativa de Irituia existe una mezcla de producción agroecológica, mixta y convencional, lo que implica que los avances en materia de sostenibilidad sean menores, en parte por la falta de asistencia técnica para que los agricultores consigan hacer la transición desde un sistema productivo convencional o mixto hacia uno agroecológico. Además, solamente una parte de los agricultores han conseguido que sus productos sean certificados por medio de OCS.

10.- El sistema de garantía de la Red Ecovida de Agroecología en el sureste de Brasil se define como un proceso para desarrollar la credibilidad a través del compromiso y la participación entre los agricultores, técnicos y consumidores con el interés común en asegurar la calidad final del producto y el proceso de producción.

11.- Símbolo del Real brasileño. En octubre de 2019 el cambio es de 0,22 euros. 


\section{Tabla 3. Criterios aplicados a ESS}

\begin{tabular}{|c|c|c|c|c|}
\hline Criterio & Dimensión analizada & Indicadores & $\mathrm{AECIA}$ & Irituia \\
\hline 7 & $\begin{array}{l}\text { Relación con los consumi- } \\
\text { dores }\end{array}$ & $\begin{array}{l}\text { (0): NS/NC; (1): inexistente; (2): indirecta; (3): poca; (4): media; } \\
\text { (5): alta. }\end{array}$ & 5 & 5 \\
\hline 8 & $\begin{array}{l}\text { Nivel de participación en re- } \\
\text { des de comercialización de } \\
\text { alimentos y de la ESS }\end{array}$ & $\begin{array}{l}\text { (0) NS/NC; (1) No; (2): informalmente en algunas redes; (3): } \\
\text { informal y formalmente, pero sin periodicidad; (4): formalmente, } \\
\text { en algunas redes con periodicidad, y en otras no; (5): sí, con } \\
\text { frecuencia y prioridad en la comercialización }\end{array}$ & 4 & 2 \\
\hline 9 & $\begin{array}{l}\text { Proceso de formación de } \\
\text { los cooperados }\end{array}$ & $\begin{array}{l}\text { (0): NS/NC; (1) no se preocupa. (2) eventual o puntualmente; } \\
\text { (3) regularmente, pero solamente con miras a la producción; } \\
\text { (4): regularmente, pero solamente con miras a la comercializa- } \\
\text { ción; (5) regularmente, en todas las áreas }\end{array}$ & 2 & 2 \\
\hline 10 & Proceso de inclusión & $\begin{array}{l}\text { (0) NS/NC; (1) muy baja. (2) baja; (3) media; (4) alta; (5) muy } \\
\text { alta -excelente. }\end{array}$ & 4 & 4 \\
\hline 11 & $\begin{array}{l}\text { Control y Participación de } \\
\text { los agricultores }\end{array}$ & $\begin{array}{l}\text { (0): NS/NC; (1): ninguna o inexistente; (2): baja; (3): media; (4): } \\
\text { alta; (5): total. }\end{array}$ & 5 & 2 \\
\hline 12 & $\begin{array}{l}\text { Relación de cooperación } \\
\text { entre los agricultores }\end{array}$ & $\begin{array}{l}\text { (0): NS/NC; (1): no hay cooperación; (2): cooperación baja; } \\
\text { (3): cooperación media; (4): cooperación alta; (5): cooperación } \\
\text { muy alta. }\end{array}$ & 4 & 3 \\
\hline 13 & $\begin{array}{l}\text { Participación en sistemas } \\
\text { participativos de garantía } \\
\text { (SPG) }\end{array}$ & $\begin{array}{l}\text { (0): NS/NC; (1): no hay participación; (2): participación baja; } \\
\text { (3): participación media; (4): participación alta; (5): participación } \\
\text { muy alta. }\end{array}$ & 4 & 3 \\
\hline
\end{tabular}

FUENTE: Nascimento, 2019.

Con relación a la ESS, en la AECIA, poseen una gran independencia en la forma de organización de los agricultores, una mayor democratización de los espacios con la presencia de las mujeres en todas las actividades de la cooperativa, aumento de la solidaridad, intercooperación tanto entre los propios agricultores como en la relación con otras cooperativas.

La ESS se está implantando de manera gradual pero aun presenta problemas de autoorganización, participación de los agricultores, interrelaciones con otras cooperativas y trabajo con mercado justo.

En cuanto a la Cooperativa de Irituia, tras el análisis realizado, podemos afirmar que comparte algunos de los principios de la ESS como, por ejemplo, la relación directa que tiene con los consumidores y la inclusión de los agricultores familiares de la región que estaban en su mayoría alejados de la comercialización de sus productos. No obstante, en otros puntos la cooperativa tiene problemas relacionados con los procesos de cooperación entre agricultores dado que más que una efectiva cooperación, se trata de relaciones puntuales de colaboración. La participación, también puntual, 
en Sistemas participativos de garantía (SPG) es otro de los principales problemas ya que muchos agricultores se integran en la cooperativa más como un medio para poder disponer del certificado de producción orgánica, que como una estrategia global de gestión de su propiedad. Por otra parte, la efectiva falta de participación en redes de comercialización de alimentos y de la ESS es otro de los principales retos, ya que esta falta de colaboración implica que esta cooperativa no participe de las ventajas del contacto con otras experiencias de ESS.

Finalmente, destacamos la problemática en materia de formación, participación y control de los agricultores en la cooperativa. Los datos demuestran que además de los anteriores, son los principales problemas relacionados con la adecuada adopción de la ESS. En la actualidad, gran parte de los agricultores consideran la cooperativa únicamente como un canal de ventas de sus productos y no como un sistema alternativo que puede enriquecer y mejorar su actividad productiva desde un punto de vista social, ambiental y económico.

\section{Conclusiones}

Este trabajo ofrece una aproximación novedosa a la sistematización y evaluación de prácticas agroecológicas, al ofrecer un enfoque donde la ESS se complementa y realiza aportes sustanciales, entre ellos: la valoración de procesos cooperativos en el territorio, la conformación de nuevos mercados con énfasis en la sostenibilidad social y económica, así como cuestiones de equidad y de género. Agroecología y ESS pertenecen al ámbito de las "economías invisibilizadas" frente a la prevalencia de la economía convencional capitalista. Sin embargo, como prueban las experiencias analizadas, son enfoques y prácticas que nos permiten organizar el metabolismo y la satisfacción de necesidades en un territorio de manera sostenible y viable, en sus planos ambiental y social. En definitiva, los enfoques integrados de estas economías que apuestan por una cooperación social ligada a los territorios constituyen alternativas al vuelco climático, la insostenibilidad derivada del crecimiento exponencial de uso intensivo de materiales y energías fósiles, las desigualdades y las hambrunas del planeta.

La construcción de canales cortos de comercialización con este acento en la ESS se revela en Brasil, como en otros países del mundo, fundamental para sostener un tejido rural vivo, que ahuyente el fantasma de la despoblación y nos acerque también a los sistemas de gestión sostenibles que propugna la agroecología.

Entre los problemas observados destacamos la creciente convencionalización del sector de la agricultura ecológica. Ello impide el avance de estrategias de desarrollo endógeno y del fortalecimien- 
to democrático de organizaciones productoras. Las decisiones quedan lejos, en manos de empresas globales y gobiernos renuentes a apoyar economías comunitarias. Los saltos de escala en el propio territorio, buscando alianzas con personas consumidoras o con otras iniciativas económicas de la zona, son frágiles. No obstante, cuando se producen, acaban favoreciendo el desarrollo de iniciativas más asentadas y encaminadas hacia la satisfacción sustentable de necesidades por parte de las personas que habitan un territorio.

De esta manera, los circuitos cortos, aproximados desde una mirada conjunta de la Agroecología y la ESS, se ofrecen como un paraguas para la democratización de nuestros sistemas agroalimentarios, a la vez que una defensa del derecho a una alimentación y una producción sostenibles, saludables y apropiadas al contexto físico y cultural.

Nuestro objetivo es continuar analizando, por un lado, las sinergias existentes entre las tradiciones científicas de la Economía Social y Solidaria y la Agroecología. Y, por otro lado, implementar propuestas metodológicas participativas que permitan comprender y acompañar experiencias concretas 0 iniciativas de políticas públicas.

\section{Referencias bibliográficas}

ALONSO, L.E. y PIÑERO C. (2015): "Presentación: el procomún y los bienes comunes", Dossieres EsF, 16, 4-7.

AGUIAR, S.D. (2009). Práticas cooperativas em redes de economia solidária sob a óptica da ação comunicativa: 0 caso da Rede Justa Trama. Universidade Fortaleza.

ALONSO, L.E. y BENITO, L.E.A. (1998): La mirada cualitativa en sociología: una aproximación interpretativa, Vol. 218, Editorial Fundamentos.

ALTIERI, M.A. (1989): Agroecologia: as bases científicas da agricultura alternativa, Rio de Janeiro: PTA/FASE.

APARECIDA DE ALMEIDA, R., GALLAR, D. \& CALLE, Á. (2014): "A 'nova' questão agrária em Andalucía: processos de recampesinização em tempos de impérios agroalimentares", Revista NERA (Núcleo de Estudos, Pesquisas e Projetos de Reforma Agrária - UNESP), 24, 9-35.

BAREA, J. (1990): "Concepto y agentes de la economía social", CIRIEC-España, Revista de Economía Pública, Social y Cooperativa, 8, 109-117. 
BIALOSKORSKI, S. (2004): "Gobierno y papel de los cuadros directivos en las cooperativas brasileñas: estudio comparativo", CIRIEC-España, Revista de Economía Pública, Social y Cooperativa, 48, 225-241.

BUAINAIN, A.M., ROMEIRO, A.R. \& GUANZIROLI, C. (2003): "Agricultura familiar eo novo mundo rural", Sociologias, 5(10), 312-347.

BYE, P. \& SCHMIDT, W. (2001): "Agriculture familiale au Sud du Brésil; d'une exclusion productiviste à une exclusion certifiée", Communication au XIXieme Congrès de la Société européenne pour la sociologie rurale, Dijon, 3-7.

CABANES, M. y GÓMEZ, J.D. (2014): "Economía social y Soberanía Alimentaria. Aportaciones de las cooperativas y asociaciones agroecológicas de producción y consumo al bienestar de los territorios", CIRIEC-España, Revista de Economía Pública, Social y Cooperativa, 82, 127-154.

CALLE-COLLADO, Á. y CASADEVENTE, J.L. (2015): "Economías sociales y economías para los Bienes Comunes", Otra Economía, 9(16), 44-68.

CALLE-COLLADO, Á., PADILLA, R.S. y PIÑEIRO, C. (2017): "Comunes y economías para la sostenibilidad de la vida", Rebeldías en común: sobre comunales, nuevos comunes y economías colaborativas, 15-46, Libros en Acción.

CALLE-COLLADO, Á. \& NASCIMENTO, F.S. (2018). "Uma análise da economia social solidaria em experiência de agricultura familiar: estudo de casos da COOPAECIA e da ECONORTE", Estudos do CEPE, 48, 69-87. DOI: http://dx.doi.org/10.17058/cepe.V0i48.13133.

CAPORAL, F.R. y COSTABEBER, J.A. (2004): Agroecologia: alguns conceitos e princípios, IICA. Brasilia.

CARPINTERO, Ó. y RIECHMANN, J. (2013): "Pensar la transición: enseñanzas y estrategias económico-ecológicas", Semimonografico. Pensar la transición (I).

CASARES, J. y REBOLLO, A. (2005): Distribución comercial, $3^{\text {a }}$ edición, Navarra, Thomson Cívitas.

CIDADE-BRASIL, 2019. Recuperado el 01/07/2019 de https://www.cidade-brasil.com.br/estado-riogrande-do-sul.html.

COSTA, B.A.L. y JESUS, P. de (2017): "AEconomia Solidária no Brasil: Uma trajetória de conformação enquanto movimiento social?", Revista Mundo Do Trabalho Contemporâneo, São Paulo, v.2.2, 241-264.

CUÉLLAR, P.M. y CALLE-COLLADO, Á. (2009): "Sistemas participativos de garantía: poder, democracia y Agroecología". En: I Congreso de Sociología de la Alimentación.

DAZA, E.F. y DANGLA, R.R. (2013): "El efecto de la crisis en dos sectores importantes de cooperativas valencianas. Similitudes y diferencias con sus homólogas en las sociedades de capital", CIRIECEspaña, Revista de Economía Pública, Social y Cooperativa, 79, 219-242. 
DIEESE (2015): "Informalidade na economia solidária / Departamento Intersindical de Estatística e Estudos Socioeconômicos. - São Paulo", Coleção Cadernos de Debates do Observatório Nacional da Economia e do Cooperativismo, 1.

DOS SANTOS, L.C.R., DE CAMARGO, A.C.M. y ALVES, A.R. (2017): "Empreendimento de economia solidária: Uma análise da autogestão coletiva/Nenture solidarity economy: An analysis of collective self-management", Brazilian Journal of Development, 1(1), 2-14.

FAO y IFAD (2019): "United Nations Decade of Family Farming 2019-2028", Global Action Plan, Rome.

FÓRUM BRASILEIRO DE ECONOMIA SOLIDÁRIA (2008). Recuperado el 28/11/2018 de http://fbes. org.br/2005/05/02/sobre-o-fbes/.

FRAISSE, L. (2004): "Economía solidaria y democratización de la economía", Economía social y solidaria. Una visión europea, Buenos Aires, Fundación OSDE-Universidad Nacional de General Sarmiento-Editorial Altamira

GAIGER, L.I. G. (2001): "As Organizações do Terceiro Setor e a Economia Popular Solidária", Revista Ciências Sociais Unisinos, 37(159), 103-151.

GAIGER, L.I.G. (2003): "L'Economie Solidaire au Brésil", Recherches - Revue du M.A.U.S.S., 21, 80-96.

GÓMEZ, J.D. y DE SOUZA, B.M.J. (2013): Estrategias y acciones de Desarrollo Rural a través de Cooperativas y Emprendimientos Solidarios, OfiBOOK, Alicante.

GONÇALVES, A.F. (2013): "Economía (s) solidaria (s) y políticas públicas en Brasil", Revista Gestão \& Conexões, 1(1), 44-54.

GONZÁLEZ CALO, M.I. (2017): "Autogestión, relocalización y reapropiación de los sistemas agroalimentarios y su biodiversidad ecológica y cultural: construyendo mercados sustentables para nuestros alimentos desde propuestas agroecológicas. La experiencia de Ecovalle en el Valle de Lecrín (Granada)". Recuperado el 07/03/2019 de http://hdl.handle.net/10396/15114.

GUZMÁN, G.I., GONZÁLEZ, M. y SEVILLA, E. (2000): Introducción a la Agroecología como desarrollo rural sostenible, No. 630.2745 I-61i, Madrid, ES: Mundi-Prensa.

LAVILLE, J.L. (Dir.) (1994): L'économie solidaire, Paris: Desclée de Brouwer.

LAVILLE, J. \& ROUSTANG, G. (1999): “L'enjeu d'un partenariat entre État et société civile”. In: Jacques Defourny et al., Economie sociale au Nord et au Sud, Bruxelles: Deboeck, 217-238.

LAVILLE, J.L. (2004): Economía social y solidaria, Una visión europea, Altamira, Buenos Aires.

LECHAT, N.M.P. (2002): "Economia social, economia solidária, terceiro setor: do que se trata?", Revista de Ciências Sociais, Ano 2, nº 1, junho, 123-140. 
MEIRINHOS, M. \& OSÓRIO, A. (2010): "O estudo de caso como estratégia de investigação em educação", EduSer-Revista de educação, Bragança, Portugal, 2(2).

MONTIEL, M.S. y CALLE-COLLADO, Á (2010): "Rearticulando desde la alimentación: canales cortos de comercialización en Andalucía", Patrimonio cultural en la nueva ruralidad andaluza, 258-283.

MOZAS, A. y BERNAL, E. (2006): "Desarrollo territorial y economía social", CIRIEC-España, Revista de Economía Pública, Social y Cooperativa, 55, 125-140.

NASCIMENTO, F.S.D. (2019): Sistema agroalimentario de base ecológica-El caso brasileño, Tesis Doctoral, Universidad de Córdoba. Recuperado el 7 de octubre de 2019 de http://hdl.handle. net/10396/18800.

NEVES, D.P. (2007): "Agricultura familiar: quantos ancoradouros", Geografia Agrária: teoria e poder, São Paulo: Expressão Popular, 1, 211-270.

NOBRE, N. (2015): «Economia solidaria, Agroecología y feminismo: prácticas para la autonomía en la organización del trabajo y de la vida». In: Verschuur Christine, Guérin Isabelle et Hillenkamp Isabelle (dir.), Une économie solidaire peut-elle être féministe? Homo oeconomicus, mulher solidaria, Paris, L'Harmattan, 273-94.

OROZCO, A.P. (2004): "Estrategias feministas de deconstrucción del objeto de estudio de la economía", Foro Interno, 4, 87-117.

PÉREZ DE MENDIGUREN, J.C., ETXEZARRETA, M. y GURIDI, L. (2008): “¿De qué hablamos cuando hablamos de Economía Social y Solidaria? Concepto y nociones afines", Comunicación presentada a las XI Jornadas de Economía Crítica, 27-29.

ONU (2014): La Economía Social y Solidaria y el Reto del Desarrollo Sostenible. Recuperado el 06/02/2019 de http://unsse.org/wp-content/uploads/2014/08/Position-Paper_TFSSE_Esp1.pdf.

ORTIZ, H. (2001): "Economia Solidária: hacia una nueva civilización", Programa de Economia Popular Solidária da Secretaria de Desenvolvimento e Assuntos Internacionais do Governo do Estado do Rio Grande do Sul.

SCHMITT, C.J. (2001): "Tecendo as redes de uma nova agricultura: um estudo socioambiental da Região Serrana do Rio Grande do Sul", Universidade Federal do Rio Grande do Sul-Programa de Pós-Graduação em Sociologia (Tese de Doutorado), Porto Alegre.

SCHULTZ, G. (2006): "Relações com o mercado e (re) construção das identidades socioprofissionais na agricultura orgânica". Recuperado el 01/02/2019 de https://www.lume.ufrgs.br/handle/10183/8585.

SEVILLA, E. \& WOODGATE, G. (1997): "Sustainable rural development: from industrial agriculture to agroecology". In: The international handbook of environmental sociology, Edward Elgar Publishing.

SEVILLA, E. (2011): Sobre los orígenes de la Agroecología en el pensamiento marxista y libertario, Plural editores, La Paz. 
SEVILLA, E., SOLER, M., GALLAR, D., VARA, I. y CALLE-COLLADO, Á. (2012): Canales cortos de comercialización alimentaria en Andalucía, Sevilla: Fundación Pública Andaluza Centro de Estudios Andaluces, Consejería de la Presidencia e Igualdad, Junta de Andalucía, 201(2), 1.

SILVA, M.B., CAPORAL, F.R., De MORAES, J.R. y MONZON, A.G. (2014): "Los límites y desafíos en el apoyo a formas más sustentables de agriculturas a través de la extensión rural pública en Pernambuco, Brasil", Revista de la Facultad de Agronomía, La Plata, 113(1), 73-80.

SILVA, S.P. \& CARNEIRO, L.M. (2016): Os Novos dados do mapeamento de economia solidária no Brasil: nota metodológica e análise das dimensões socioestruturais dos empreendimentos, IPEA.

SINGER, P. (2003): Globalização e Desemprego, 6 a ed. São Paulo, Contexto.

TAUILE, J.R. \& DEBACO, E.S. (2002): Autogestão no Brasil: a viabilidade econômica de empresas geridas por trabalhadores, São Leopoldo: Unisinos.

VAN DER PLOEG, J.D. (2010): "La crisis alimentaria, la agricultura industrializada y el régimen imperial", Diario de Cambio Agrario, 10(1), 98-106. 
